Vol. 4. No. 2. (26-51) Julio-Dic/2019

ISSN: 2539-1518

\title{
Educación Inclusiva: una reflexión aproximada sobre la formación del método
}

\author{
Aldo Ocampo González \\ aldo.ocampo.gonzalez@gmail.com \\ Centro de Estudios Latinoamericanos de Educación Inclusiva \\ (CELEI). Chile.
}

\section{Resumen}

Este artículo expone de forma sintética un conjunto de consideraciones analítico-metodológicas implicadas en la comprensión del objeto y método de la Educación Inclusiva ${ }^{1}$. El trabajo inicia reconociendo que ambos elementos constituyen ámbitos complejos y contingentes, propiedad de la nulidad investigativa. A pesar que el objeto ha mutado, se observa la insistencia por inscribirlo en una estructura de institucional de conocimiento falsificada. Sumado a ello, la carencia de un método ${ }^{2}$ apropiado y coherente a la base epistemológica que expresa este enfoque. En síntesis, afirmaré que su objeto ha cambiado, no así sus métodos de aproximación e investigación, reafirmando la necesidad de su construcción. No se trata de elegir el método más apropiado para estudiar la Educación Inclusiva -lo que hasta cierto punto puede resultar estéril-, sino más bien, sentar las bases de un 'método' y de una 'metodología' apropiada al enfoque aquí discutido. En un primer momento, el trabajo analiza las tensiones implicadas en la genealogía de un objeto post-disciplinario, sus conexiones y repercusiones con la construcción de su método. En un segundo momento y final, se analiza el papel de los conceptos, concebidos como herramientas epistemológicas capaces de distorsionar o bien, ayudar a comprender un determinado objeto de conocimiento. El trabajo concluye profundizando en los conceptos que permiten estudiar la Educación Inclusiva como una ciencia post-disciplinar.

\section{Palabras clave}

Objeto post-disciplinar, método no creado, apertura de la estructura disciplinaria, inclusión y conceptos para leer el presente.

Recibido 17/08/2019* Aceptado 15/10/2019.

\footnotetext{
1 Proyecto epistémico y metodológico crítico complejo.

2 En adelante déficit metodológico.
} 


\title{
Inclusive Education: an approximate reflection on the formation of the method
}

\begin{abstract}
This article summarizes a set of analytical-methodological considerations involved in the understanding of the object and method of Inclusive Education. The work begins recognizing that both elements constitute complex and contingent areas, property of the investigative nullity. Although the object has mutated, the insistence is observed for inscribing it in an institutional structure of falsified knowledge. Added to this, the lack of an appropriate and coherent method to the epistemological base that expresses this approach. In synthesis, I will affirm that, its object has changed, not so, its methods of approach and investigation, reaffirming the necessity of its construction. It is not about choosing the most appropriate method to study Inclusive Education -which can be sterile to some extent-, but rather, laying the foundations of a 'method' and a 'methodology' appropriate to the approach discussed here. At first, the work analyzes the tensions involved in the genealogy of a post-disciplinary object, its connections and repercussions with the construction of its method. In a second and final moment, the role of concepts is analyzed, conceived as epistemological tools capable of distorting or helping to understand a certain object of knowledge. The work concludes by deepening the concepts that allow to study Inclusive Education as a post-disciplinary science.
\end{abstract}

\section{Keywords}

Post-disciplinary object, method not created, opening of the disciplinary structure, inclusion and concepts to read the present. 


\section{Educação Inclusiva: uma reflexão aproximada sobre a formação do método}

\section{Resumo}

Este artigo resume um conjunto de considerações analítico-metodológicas envolvidas na compreensão do objeto e método da Educação Inclusiva. O trabalho começa reconhecendo que ambos os elementos constituem áreas complexas e contingentes, propriedade da nulidade da investigação. Apesar de o objeto ter sofrido uma mutação, observa-se a insistência em inscrevêlo em uma estrutura institucional do conhecimento falsificada. Somado a isso, a falta de um método apropriado e coerente à base epistemológica que expressa essa abordagem. Em resumo, afirmo que seu objeto mudou, mas não seus métodos de abordagem e investigação, reafirmando a necessidade de sua construção. Não se trata de escolher o método mais apropriado para estudar a Educação Inclusiva - que até certo ponto pode ser estéril -, mas sim, lançar as bases de um 'método' e uma 'metodologia' apropriados à abordagem discutida aqui. Inicialmente, o artigo avalia as tensões envolvidas na genealogia de um objeto pós-disciplinar, suas conexões e repercussões na construção de seu método. Num segundo e último momento, analisa-se o papel dos conceitos, concebidos como ferramentas epistemológicas capazes de distorcer ou ajudar a entender um determinado objeto de conhecimento. $\mathrm{O}$ trabalho conclui aprofundando os conceitos que permitem estudar a Educação Inclusiva como ciência pós-disciplinar.

\section{Palavras-chave}

Objeto pós-disciplinar, método não criado, abertura da estrutura disciplinar, inclusão e conceitos para a leitura. 


\section{1.-Introducción: implicancias epistemológicas sobre Educación Inclusiva}

La Educación Inclusiva ${ }^{3}$ constituye un campo teórico compuesto de múltiples entradas a la comprensión de su objeto, así como una teoría sin disciplina. Devenida en una estructura de conocimiento que se nutre de aportes de diversa naturaleza -prácticas heterogéneas-, recurre a la ecología de saberes -concebida como un sistema de reciclaje epistemológico y un mecanismo de interconexión de una multiplicidad epistemológica infinita de saberes-, al examen topológico -sistema analítico mediante el cual es posible graduar los niveles de proximidad y transferencia de recursos metodológicos en la confluencia de dos o más campos del conocimiento- y a la traducción, configura un saber que desborda las programaticidades tradicionales legitimadas por las estructuras disciplinares ${ }^{4}$. La expresión 'sin disciplina' ${ }^{5}$ alude a la cualidad viajera de la Educación Inclusiva, esto es, su capacidad para movilizarse a través de una amplia confluencia de discursos, métodos, objetos, teorías, disciplinas, compromisos éticos, proyectos políticos e influencias, describiéndolo como un campo no-fijo en ninguna disciplina. ¿Cómo llega a ocupar esta posición?

En los últimos años el concepto de inclusión ha designado un terreno fundamentalmente disputado por una multiplicidad de disciplinas académicas, discursos, concepciones políticas y corrientes críticas más prominentes de nuestra época. ¿Cómo llega a ocupar esta posición? Ofrecer una respuesta a interrogantes de tales características, sugiere emprender una crítica genealógica sobre el conjunto de condiciones sociales, institucionales e intelectuales que propician su objeto de conocimiento. Uno de sus propósitos consistirá en cuestionar los diversos tipos de relaciones que tienen lugar a través de cada uno de los elementos que dan vida al campo de estudio de la Educación Inclusiva. Coincidiendo con Knorr (2005) y Mowitt (1992), es menester profundizar en el estudio de la lógica cultural disciplinaria que permea su funcionamiento y campo de acción. Sugiere, además, develar los problemas postdisciplinarios que este enfrente en su desarrollo.

\footnotetext{
${ }^{3}$ Constituye una práctica teórica y metodológica abierta, compleja y de intensidad inestable.

4 Constituye una cita y un comentario limítrofe y fronterizo en la examinación de la realidad. También puede ser lecturada como una estrategia de lo refractario.

${ }^{5}$ Reafirma su carácter post-disciplinar. Construye su conocimiento movilizándose por diversas disciplinas, métodos, objetos, territorios, discursos, saberes, conceptos, compromisos éticos y proyectos políticos. Constituye un campo atravesado y disputado fundamentalmente por un conjunto de prácticas teóricas, metodológicas, analíticas y discursivas de carácter complejas.
} 
El espacio de diáspora ${ }^{6}$ no solo constituye un mecanismo de enmarcamiento que alberga un conjunto heterogéneo de elementos que circulan en su interior en diversas direcciones, describe las problemáticas del movimiento que afectan a cada uno de sus elementos confluyentes en él, las disciplinas se movilizan permanentemente. Se encuentra caracterizado por la transmigración de saberes, la rotación, el movimiento y la interseccionalidad como condición de producción. En su interior tienen lugar diversos cruces y mecanismos de transgresión de las fronteras de cada una de sus disciplinas y campos de confluencia. Conceptos significativos en la comprensión del espacio epistemológico de la Educación Inclusiva son: a) experiencia migratoria, b) diáspora epistémica, c) políticas de localización de saberes multiaxial, d) enredos genealógicos de dispersión, e) diálogo, interconexión, mediación epistémica, etc. De esta forma, su campo de conocimiento queda definido por su condición de heterotopicalidad, es decir, confluencia de tópicos de análisis de diversa naturaleza, sintonizados coherentemente hacia su estructura de conocimiento. Es menester señalar que la Educación Inclusiva no suscribe a ninguna práctica teórica y metodológica en particular, a pesar de enfrenar el desafío de encontrar su propio método.

El pensamiento epistemológico de la Educación Inclusiva es el 'pensamiento de la relación' (dispositivo-ensamblaje), del 'movimiento epistémico de saberes, conceptos $y$ métodos' (diáspora epistémica) y del 'encuentro' (zona de contacto y producción de lo nuevo). El ensamblaje y su arquitectura epistémica, operan mediante la movilización de las fronteras de las disciplinas, confluyendo en una zona de contacto, cuya interrelación fabrica una 'frontería epistémica', es decir, una zona de indeterminación, de apertura, de creación, de un tercer espacio, denominado 'heterotópico"7, coherente con su naturaleza post-disciplinar.

\footnotetext{
${ }^{6}$ Espacio concebido en termino de relaciones diaspóricas, ahí que Ocampo (2016) propusiera la metáfora: 'el conocimiento de la Educación Inclusiva se construye diaspóricamente'. Lo diaspórico en la construcción epistemológica de la Educación Inclusiva se convierte en un terreno de esperanza, de encuentro con lo diferente, forma una malla de conexión de elementos infinitamente heterogéneos entre sí, establece múltiples rutas, prevalece el sentido de lo no-idéntico. Un espacio diaspórico de producción en la Educación Inclusiva otorga a cada objeto, método, disciplina, discurso, sujeto, territorio, teoría, influencia, compromiso ético, marco teórico y proyecto político una posición clara, al mismo tiempo móvil.

${ }^{7}$ Espacio libre de actuaciones de los formatos del poder.
} 


\section{2.-Educación Inclusiva: la configuración de un objeto ${ }^{8}$ post-disciplinario}

Sin duda la pregunta por el 'objeto' sigue siendo uno de los ámbitos analíticos más complejos e ininteligibles -e incluso, no abordados por los investigadores- junto al 'método' en el contexto de la Educación Inclusiva. Una primera aproximación consistiría en reconocer la complejidad que atraviesa al 'objeto', 'método' y 'campo', pues estos, son precedidos por una nebulosa que afecta a la comprensión de la naturaleza de su estructura teórica, caracterizada por leyes del pensamiento que conducen a un efecto de 'falsificación' e 'hibridación'9 de sus principales cuerpos de saberes, instrumentos conceptuales y herramientas metodológicas. A pesar que el objeto ha mutado, se observa la insistencia por inscribirlo en una estructura de institucional ${ }^{10}$ de conocimiento falsificada. Sumado a ello, la carencia de un método apropiado y coherente a la base epistemológica que expresa este enfoque. En síntesis, afirmaré que, su objeto ha cambiado, no así, sus métodos de aproximación e investigación, reafirmando la necesidad de su construcción. No se trata aquí de elegir el método más apropiado para estudiar la Educación Inclusiva -lo que hasta cierto punto resulta estéril, sino más bien, sentar las bases de un método y de una metodología que emerja en sintonía con su base epistemológica. Su creación proporciona herramientas acertadas para efectuar una aproximación certera a su objeto y estructura de conocimiento. Método y objeto determinan la pertinencia de la intervención.

En efecto, su interés fundacional consiste en el develamiento de nuevas formas intelectuales y metodológicas que contribuyan a interrogar la realidad, los modos de pensar, experimentar y practicar la Educación Inclusiva, desde la cristalización de nuevas espacialidades -labor positiva-, así como, estrategias que contribuyan a movilizar nuevas racionalidades capaces de problematizar la escolarización, el desarrollo ciudadano y político, interrogar acerca de las posibilidades que proporcionan

\footnotetext{
${ }^{8}$ Es un objeto intersticial que emerge de una red de segmentaciones dispersas, proliferando un nuevo contexto de referencialidad. "Un entre-lugar fluctuante que, al surgir de las discontinuidades y variaciones de lo global, nunca logra autoafirmarse naturalmente como una territorialidad satisfecha que se concibe propietaria de sí misma" (Richard, 2009, p.27). se encuentra en permanente construcción, da paso a una modalidad teórica de intensidad inestable, que frente a diversos fenómenos va irrumpiendo hacia otras lógicas. Describe también una acción cognitiva de la novedad, de la irrupción de lo nuevo, una operación tropológica que gira los significados, sentidos y alcances hacia otras comprensiones.

${ }^{9}$ Lo hibrido se emplea para designar las mezclas de fragmentos que circulan translocalizadamente, forjando prácticas articulatorias que tienen lugar en repertorios epistémicos discontinuos.

10 Concibo lo institucional como el saber dominante y legitimo al interior de las estructuras académicas dedicadas a la formación y a la investigación en el campo de la Educación Inclusiva.
} 
los marcos y vocabularios existentes, ante la heterotopicalidad de tensiones analíticometodológicas que atraviesan su campo de producción.

El efecto de falsificación ${ }^{11}$ epistemológica se expresa por la presentación de argumentos, abducción desmedida de conceptos y significados y saberes que no se encuentran en relación con la naturaleza del conocimiento de la Educación Inclusiva, a pesar de vincularse en algún eje de tematización, constituyen puntos ciegos en la formulación de su saber. Por lo general, las premisas y expresiones de la falsificación tienden a la desvirtuar el interés por la comprensión auténtica de su objeto ${ }^{12}$, distorsionando su sentido y alejando la ubicación de su actividad científica. Para Mowitt (1992) la falsificación epistemológica puede ser lecturada en términos de una expresión del paradigma dominante de una época, estipulando condiciones de aceptación, a pesar que pueda diferir del paradigma de base. La falsificación en este contexto se expresa por medio de la sustitución de saberes y conceptos -concebidos estos últimos desde la perspectiva de Bal (2002) como teorías en miniaturas- abducidos desde la imposición epistémica y didáctica de la Educación Especial -en su dimensión pedagógica-, reforzando con ello, un marco de valores atemporal y mutilado para referir al estudio de los procesos de inclusión y exclusión, insistiendo en el individualismo metodológico y en la construcción de una espacialidad política, pedagógica e imaginativa sustentada en la abyección -problema ontológico de los grupos sociales-. Entonces, ¿por qué se produce tal falsificación?, básicamente, debido a la ausencia de una construcción teórica y epistemológica, se procedió a justificar sus sistemas de razonamientos desde el campo antes citado. Esto es lo que hace que los investigadores se aproximen erróneamente a la comprensión de su objeto, constituyendo el paradigma dominante dentro de un determinado marco conceptual ${ }^{13}$.

En mi opinión, a partir de la visibilización de tópicos de diversa naturaleza que configuran sus ámbitos de discusión y, específicamente, de la propiedad extradisciplinar que expresa la configuración y funcionamiento de su campo de conocimiento y producción -específicamente, esta última propiedad-permite observar un conjunto de elementos responsables de la apertura de su estructura disciplinaria. Tal situación

\footnotetext{
${ }^{11}$ Véase "La enseñanza de la Educación Inclusiva y la pregunta por el método: un análisis antidisciplinar".

${ }^{12}$ Hacen parecer que la teoría es algo existente, disponible y construida.

${ }^{13}$ Los conceptos empleados actualmente tienden a desvirtuar y la distorsionar la comprensión del objeto. A este efecto epistemológico posicionado he denominado tropismo negativo.
} 
desafía el dogma metodológico, interrogando los límites disciplinarios. Son estos los elementos, que permiten atender a la formación/mutabilidad de un tipo diferente de objeto, caracterizado como un espacio contrario a los enfoques tradicionales e incluso, desgastado producto de la crisis de representación.

Las fuerzas de hibridación en tanto recurso analítico, pueden describirse en términos de mecanismos de modelización del campo, formas de devenir de las disciplinas y sus conceptos. En actualidad, el campo de conocimiento de la Educación Inclusiva, al reconocer que esta, no es educación Especial, en su dimensión pedagógica $y$, producto del repertorio de tecnologías extra-disciplinares que contribuyen en su funcionamiento -herramientas que permiten conectar campos alejados en su actividad científica, pero vinculados de algún modo al objeto de la inclusión-, se observa la apertura de su estructura disciplinaria producto de la convergencia y confluencia de disciplinas tales como, los Estudios del Feminismo, de la Mujer, Queer, Post-coloniales, la Filosofía Política y de la Diferencia, entre otras. El campo hibrido y mixto de la Educación Inclusiva se compone del entrecruzamiento de diversas herramientas metodológicas, saberes y conceptos, abducidos arbitrariamente, acompañados de la técnica de aplicacionismo epistemológico, recurre a tomar prestados conceptos y herramientas metodológicas procedentes de diversas disciplinas, atrapando el saber en sus marcos disciplinarios. Todo ello, devela un enredo de recursos metodológicos que requieren de la búsqueda de sistema de traducción científica y epistemológica, a fin de superar las prácticas de aplicacionismo epistemológico.

¿A raíz de la participación de qué disciplinas, discursos, campos del conocimiento y conceptos, la estructura disciplinaria de la Educación Inclusiva ha sido objeto de apertura hacia otras formas comprehensivas? Si bien es cierto, la estructura de conocimiento $^{14}$ de la Educación Inclusiva se articula por fuera de los límites disciplinarios, específicamente, en la intersección de dos o más disciplinas que en ella confluyen -es una teoría sin disciplina-, se propone construir un nuevo saber exterioridad epistemológica-, que emerge de la extracción de los aportes más significativos de cada disciplina, objeto, teoría, influencia, marco teórico y político, discursos y territorios por los que esta se moviliza. Es un saber en permanente movimiento y transformación. No obstante, requiere de condiciones de traducción,

\footnotetext{
${ }^{14}$ Compuesta por un conjunto de prácticas teóricas y metodológicas heterogéneas.
} 
examinación topológica y ecología de saberes -elementos claves en la producción de los saberes auténticos del enfoque-. La configuración de un espacio de producción diaspórico y de una red reticular que alberga elementos de diversa naturaleza y textura, exige metodológicamente condiciones de traducción de cada uno de sus principales elementos confluyentes. La Educación Inclusiva es, sin duda alguna, un terreno fundamentalmente disputado por una multiplicidad de disciplinas académicas, discursos, concepciones políticas y corrientes críticas más prominentes de nuestra época.

Si bien es cierto, el objeto de la Educación Inclusiva en la literatura especializada se expresa mediante connotaciones travestizadas, hibridizadas y poco claras, es plausible afirmar que, su objeto se encuentra en constante movimiento y, por tanto, no le pertenece a nadie como tal, a pesar de enfrentar el desafío de develar sus condiciones de autenticidad y crear un nuevo objeto y saber. Razón por la cual, afirmaré que, a partir de la incorporación discursiva de la 'totalidad' -concebida en bloque- el objeto aquí debatido comienza a descentrarse y toma distancia de los supuestos analítico-metodológicos que lo inscriben erróneamente en una regulación discursiva propiedad de la abyección y del individualismo metodológico. Se observa de este modo, un conjunto de debilidades que permitan identificar las principales innovaciones que este campo ha experimentado silenciosamente en el abordaje de su objeto, es decir, prestar atención a un tipo diferente de objeto, contrario a los enfoques y sistemas de razonamientos tradicionales. Esta identificación ha sido infértil, pues, gran parte de la investigación sobre Educación Inclusiva continúa inscribiendo su actividad en ámbitos de falsificación epistemológica ${ }^{15}$. Una de las causas que explica porque la atención a un nuevo objeto de conocimiento no ha tenido un éxito suficiente, se debe a la incapacidad para reconocer que métodos de aproximación, abordaje e investigación permitan asumir tal empresa. Sumado a ello, el conjunto de errores de aproximación al objeto por parte de los investigadores. En cierta media, esta empresa no ha tenido un éxito suficiente, producto que ha sido incapaz de contrarrestar los métodos de exclusión de sus diversas disciplinas concursantes. Todo ello demuestra que, los métodos no han cambio incluso, si me propongo ser más radical, sería oportuno mencionar que dichas debilidades se desprenden de la ausencia de una construcción metodológica coherente

15 Principal efecto de las prácticas de investigación, formación del profesorado y de las políticas educativas. 
con su estructura y naturaleza epistemológica-. Lo único que ha mutado es aquello que estudiamos, es decir, el objeto de la Educación Inclusiva, en términos de una ficción regulativa de tipo imaginativa, es decir, se observa un breve desplazamiento retórico, no así, soportes teóricos y metodológicos que puedan acompañar dicha empresa. La deuda sigue expresando el cómo. Debido a la ausencia de un método -el cómo hacemos- ha sido resuelto mediante una combinación inadecuada de diversos recursos metodológicos -situando la respuesta en la fijeza metodológica heredada-, lo cual, ha restringido la pregunta por los elementos que justifican los ámbitos de constructividad del método.

Retomando la interrogante acerca del objeto de la Educación Inclusiva, quisiera plantear la siguiente interrogante: ¿dónde se encuentra el objeto?, el interés por las coordenadas de ubicación del fenómeno nos permite aclarar un conjunto de errores y comprensiones distorsionadas; incluso, identificar y transformar aquellos conceptos que contribuyen a desvirtuar su objeto ${ }^{16}$. Una aproximación inicial consistiría indagar acerca del tipo de 'educación' al que nos conduce su objeto de conocimiento. Es, este punto, el que jamás se menciona en los debates más críticos sobre Educación Inclusiva. La pregunta por el objeto es determinante, puesto que, permea las características ontológicas, epistemológicas y metodológicas ${ }^{17}$ del fenómeno, delimita los parámetros de acción de su universo conceptual y traza las rutas metodológicas para su concreción. Retomando la pregunta, ¿dónde se encuentra el objeto?, es decir, éste, inscribe su actividad en la educación o en la inclusión. La parte de 'educación' es lo que está en juego del nombre de Educación Inclusiva, ¿qué elementos constituyen su objeto de análisis? La pegunta que planteo en este párrafo, debe ser considerada como fundamental, ofrece claves para acceder a una comprensión situada sobre su naturaleza. En diversas clases y conferencias donde he abordado la pregunta por el objeto, solicito a los participantes que efectúen una lluvia de ideas de mínimo diez palabras y máximo diez palabras, respecto de aquellas cosas o significados que, para ellos, se encuentran directamente vinculadas con la Educación Inclusiva. Al efectuar la puesta en común, las respuestas son, en su mayoría; educación para todos, educación orientada a superar los efectos opresivos y las trabas en el (auto)desarrollo, su misión consiste en la ampliación de oportunidades, la creación de condiciones de justicia social, la educación de las

${ }^{16}$ Su objeto y método forjan una epistemológica posicionada.

17 Niveles de sustentación de la actividad científica. 
diferencias, respecto, tolerancia, reconocimiento del otro; en síntesis, un largo etcétera. Luego, les solicito que elijan cinco palabras o ideas más significativas para ellos. Una vez elegidas, les planteó la siguiente interrogante: ¿estas ideas, son propias de la educación o de una educación específica o especializada?, mayoritariamente, las personas responden rápidamente señalando que son intrínsecas a la tarea educativa. Mediante este sencillo ejercicio, podemos comprobar que el objeto y la naturaleza del fenómeno se inscriben en la educación. En suma, el adjetivo inclusivo, únicamente posee la capacidad de movilizar el cambio, mediante una sensibilización. En su sentido más positivo, permite reconocer qué elementos y prácticas han contribuido a diluir estas ideas del centro de la actividad educativa. Una educación inclusiva, es sustantivamente, actividad educativa a secas.

Por tal razón, la contraparte de cualquier concepto que se proponga describir el objeto de la Educación Inclusiva, converge en lo educativo. Es esto lo que constituye su objeto. Tal como indica Bal (2003) el concepto solo se vuelve significativo cuando es capaz de describir y comprender mejor el objeto en sus propios términos. Así, la atención sostenida al objeto se convierte en una de las tareas críticas que enfrenta la educación. ¿Qué naturaleza poseen los objetos de estudios de las disciplinas que confluyen en el espacio de producción de la Educación Inclusiva?, el problema es que no todos ellos estudian la educación, más bien, se conectan en algún punto al fenómeno, pero no estructuran su campo de actividad a partir de ello. Aquí encontramos los casos de la Filosofía, la Antropología, la Sociología, por ejemplo. Este análisis no restringe su concepción a una práctica o concepción particular de educación. ¿Qué rol cumple el calificativo 'inclusiva' en Educación Inclusiva? Una posible consideración consistiría en movilizar una distinción sobre la práctica disciplinaria tradicional al interior de la Ciencia Educativa. Mediante su prisma post-disciplinar, el análisis de la multiplicidad de objetos que en ella confluyen se asume como elementos integrados y comprometidos con objetivos más amplios, orientados a la construcción de un nuevo marco teórico y conceptual.

También significa que su análisis se enfoca en temáticas de relevancia educativa, articulando contribuciones significativas para develar su capacidad de enriquecer los debates y las intervenciones educativas contemporáneas y con ello, transformando sus vocabularios existentes. Esto es, lo que hace que la existencia del objeto se encuentre en 
el presente. ¿Cómo participa este objeto en la producción de significados educativos? La interrogante acerca del tipo de significados que construye el objeto en relación a los debates educativos, contribuiría en cierta medida a responder la pregunta acerca del tipo de educación -sus lenguajes y modalidades de escolarización- y praxis social a la que conduce dicho fenómeno. ¿A qué tipo de análisis sobre las problemáticas y fenómenos educativos nos conduce este objeto y qué herramientas metodológicas se sugieren para ello?

Tal como ha afirmado en trabajos anteriores, la naturaleza del conocimiento de la Educación Inclusiva presenta un carácter post-disciplinar, es decir, construye un nuevo saber que emerge de los intersticios de cada una de sus disciplinas confluentes, explicitando un estilo específico de reunión y re-articulación. En este marco, es posible afirmar que, esta, presenta un objeto de conocimiento complejo y multidimensional de naturaleza anti/post-disciplinar y fronteriza, a pesar de inscribir su fuerza teórica en la educación. En los párrafos que se exponen a continuación, me propongo abordar algunas de las principales complejidades que expresa el objeto anti/post-disciplinario ${ }^{18}$ de la Educación Inclusiva. Como puntapié introductorio, afirmaré que, este constituye un objeto complejo, atravesado no-linealmente por elementos e influencias de diversa naturaleza y textura, compuesto por mecanismos particulares de enmarcamiento desafiando los dogmas metodológicos legitimados. Como tal, puede ser lecturado como un terreno fundamentalmente disputado dentro de una serie de disciplinas académicas. Resultando imperioso develar la forma en que llegó a ocupar dicha posición y el tipo de tropismos que de él se desprenden, específicamente; cuando nos preguntamos acerca del tipo de educación, sociedad, escolarización y sujeto al que nos conduce. El reconocimiento de un objeto anti/post-disciplinario -como el aquí analizado- exige un examen sobre la genealogía crítica de las condiciones sociales e intelectuales que propenden a su emergencia, inaugurando una invitación para repensar las condiciones de producción de la Educación Inclusiva. Enfatizar en una dimensión genealógica de su objeto, sugiere identificar tensiones heterotopicales y problemáticas anti/postdisciplinares que van definido un particular contexto de emergencia.

Una exploración post-disciplinaria sobre objeto de la Educación Inclusiva nos remite a la interrogante sobre los elementos de dislocación de su significado. La

${ }^{18}$ Lo anti-disciplinario y lo post-disciplinario expresan un carácter de parasinónimos. 
comprensión acertada de su objeto fortalece el compromiso con la teoría. En este encuadre, objeto y método deben ser escritos e inventados, su espacio de producción se convierte en un lugar conflictivo. Su dimensión anti/post-disciplinar, opera mediante condiciones de re-apropiación, redefiniendo sus formas de transferencia. Altera la forma en que sus investigadores y profesionales se comprometen en el abordaje del fenómeno, así como, la cristalización de sus prácticas y sus formas de constructividad, mediante condiciones de re-apropiación. Se convierte en un campo de conexión de historicidades. Parafraseando a Mowitt (1992) la fabricación de este objeto se adentra en la elaboración de una crítica profunda a la razón disciplinaria.

La construcción del objeto de la Educación Inclusiva concebido como una genealogía anti/post-disciplinaria, atiende a la potencialidad analítica del concepto de ‘posición’ y ‘transferencia', respecto de la comprensión del conjunto de articulaciones y re-articulaciones que experimentan determinados saberes, conceptos, objetos, discursos y disciplinas en la red reticular que alberga la configuración de su campo de conocimiento. Al inscribir el objeto en una perspectiva anti/post-disciplinaria, se observa cómo cada disciplina, influencia, teoría, marco teórico y político, discurso y objeto es capturado y re-articulado mediante un sistema de traducción científica. En palabras de Mowitt (1992) un examen de esta naturaleza, exige una discusión más amplia en torno a la razón disciplinaria y a sus formas de legitimación y poder que en ella tienen lugar. Esto nos contribuye a la identificación acerca de cuál es la idea de educación que se pone en juego a través de su genealogía post-disciplinar, mediante la cual, la multiplicidad de elementos que configuran su campo de producción -diaspórico y reticular- son re-articulados mediante condiciones específicas, exclusivamente estipuladas por la naturaleza del campo y de su orden de producción ${ }^{19}$. Lo sugestivo de la comprensión epistemológica de la Educación Inclusiva consiste en reconocer que su objeto inscribe su actividad como el resultado de complejas, intensas e interactivas condiciones de mediación entre diversas disciplinas y singularidades/recursos epistemológicos heterogéneos. Es un objeto ${ }^{20}$ en permanente movimiento, por lo que podría describirse como un fenómeno itinerante, que articula su actividad en las

19 El orden de producción de la Educación Inclusiva se articula y cristaliza en una operación dispórica y diseminal.

${ }^{20}$ A pesar de encontrarse en permanente movimiento, su formación se articula al interior de una formación social particular. 
intersecciones de las disciplinas, viajando no-linealmente entre ellas, cuya principal propiedad epistemológica es la multiplicidad infinita de elementos. Al situar el objeto no-fijo en un marco estrictamente disciplinario, queda vinculado a un momento histórico particular - validando una gramática particular y modos de acceso a dicho objeto- exige describirlo como una estrategia de intervención crítica decisiva en un determinado encuadre cultural. Es, un fenómeno en el que se tejen y entrecruzan discusiones de diversa naturaleza.

Un aspecto metodológico crucial consiste en develar cómo son abordadas en él, las propiedades y los límites de los fenómenos que comprenden su dominio, girando la discusión hacia una examinación estructural implicada en la configuración institucional de su conocimiento. Como tal, este es un objeto recuperado de una confluencia especifica de disciplinas y otros recursos, los que son capturados mediante tecnológicas específicas -mapeando las causas de este tipo de confluencia-. ¿Con qué se asocia la inclusión en su dominio tradicionalmente aceptado?, esencialmente, inscribe su fuerza en la sustitución de los legados de la Educación Especial, restringiendo la comprensión estructural que atraviesa la producción de este fenómeno. Su formación demanda la comprensión del tipo de operaciones que tienen lugar al interior de cada uno de sus recursos confluyentes y, específicamente, como sugiere Mowitt (1992), es necesario efectuar una crítica que permita establecer un conjunto de distinciones analíticas y metodológicas en torno a las condiciones que habilitan la participación de cada una de las disciplinas y otros recursos epistemológicos implicados en su aparición.

La comprensión de un objeto post-disciplinario plantea el desafío teóricopráctico de develar los procedimientos y herramientas a través de los cuales, es posible, reescribir el carácter de la Educación Inclusiva como un término crítico e irreductiblemente anti-disciplinario, intentando demostrar cómo es re-escrito el fenómeno a través de esta óptica. El encuadre anti/post-disciplinario es, en sí mismo un mecanismo de re-articulación del fenómeno educativo, de las prácticas de investigación y de los vocabularios existentes. Un aspecto interesante documento por Mowitt (1992) respecto de la formación de los objetos anti-disciplinarios, consiste en la articulación de condiciones para ubicar la comprensión del fenómeno en la luminosidad epistemológica, esto es, superar las prácticas científicas anquilosadas, que únicamente contribuyen a encapsular el fenómeno en sus marcos disciplinarios, restringiendo la 
comprensión por la naturaleza del mismo. En tal caso, Mowitt (1992) indica que los objetos disciplinarios, no necesariamente son reales, más bien, devienen en la constitución de una ficción regulativa, orientando la investigación dentro de un campo particular. En la actualidad, los ámbitos de inteligibilidad sobre el objeto de la Educación Inclusiva, demuestran un nulo desarrollo, a pesar de este, haber cambiado, respecto de las ideas fundacionales del movimiento, se constata la inexistencia de una matriz institucional capaz de albergar dichos planteamientos.

\section{3.-Conceptos para el estudio de la Educación Inclusiva: algunas aproximaciones}

El estudio de los conceptos constituye una empresa fundamental en el trabajo epistemológico. Los conceptos se convierten en elementos organizativos de la realidad. Según Bal (2002), estos, constituyen herramientas intersubjetivas que facilitan la comprensión y el entendimiento entre diversos campos y geografías del conocimiento. En esta perspectiva, los conceptos pueden ser concebidos como estrategias de intermediación. El estudio de los conceptos de la Educación Inclusiva constituye otro ámbito analítico no abordado. Si bien, estos pueden ser estudiados en relación al corpus de elementos que definen la naturaleza de su universo conceptual, tal como he señalado en trabajos anteriores, 'universo conceptual' y 'gramática' en este caso, son propiedad de la multiplicidad, es decir, conceptos como diferencia, diversidad, heterogeneidad, alteridad, otredad, entre otras, emergen y se encuentran contenidas en el concepto de multiplicidad. El universo conceptual de la Educación Inclusiva, en la actualidad, organiza su actividad científica en los presupuestos del lenguaje de la abyección y del individualismo metodológico, distorsionando la comprensión de su objeto de conocimiento auténtico (Ocampo, 2018a). Los conceptos en tanto herramientas epistemológicas poseen a juicio de Bal (2002) la capacidad de fundar un campo de conocimiento - específicamente, cuando el analisita posee escasa información, tal como es el caso del enfoque aquí analizado-y describir un determinado objeto, transfieren categorías claramente definidas. El universo conceptual de la Educación Inclusiva, inevitablemente, para articular su actividad toma prestadas herramientas conceptuales de diversa naturaleza procedentes de diversos proyectos intelectuales contemporáneos, demostrando un cierto efecto de contaminación lingüística y abducción desmedida de 
conceptos. De esta forma, devienen en herramientas incapaces de organizar un fenómeno de forma clara, nueva y relevante.

Los conceptos de la Educación Inclusiva se caracterizan por poseer un estatus de flexibilidad, es decir, viajan entre las disciplinas, objetos, teorías y campos del conocimiento, transformando su sentido y alcance en cada nuevo aterrizaje. Un concepto establece condiciones de adecuación en un determinado campo de conocimiento, en la medida que es capaz de ofrecer una organización adecuada del fenómeno en análisis. Si transferimos esta afirmación al campo de producción actual de la Educación Inclusiva, es posible observar, presencia de conceptos que tienden a distorsionar su objeto de conocimiento. A esto fenómeno he denominado 'tropismo negativo' (Ocampo, 2018b), es decir, la capacidad de girar los conceptos y sus significados hacia una comprensión distorsionada e incomprensible respecto del fenómeno en análisis. La condición tropológica de la Educación Inclusiva posee la capacidad de girar las comprensiones hacia la producción de nuevos significados particularmente, hacia la re-estructuración del fenómeno-, razón por la cual, su objeto de conocimiento se encuentra en constante movimiento y en continua transformación. Los conceptos poseen la capacidad de describir específicamente un objeto, afectando la realidad y enmarcando la praxis investigativa. En tal caso, su actividad en el quehacer epistemológico deviene en una epistemología posicionada, más que en una mera proyección de ideas sueltas -situación actual de este campo, producto de las políticas de todo vale y la elasticidad del mismo, principal propiedad de su campo de conocimiento-, o bien, tal como indica Strangers (1994), cristalizan un mecanismo de aplicación ligera de conceptos, que al ser empleados de forma injustificada, deviene en prácticas infértiles para especificar algún fenómeno, es decir, ofrecer condiciones analíticas para lecturar un determinado fenómeno. Sin duda, es menester analizar las condiciones que acompañan la producción de un nuevo significado.

Los conceptos que actualmente estructuran el campo de actividad científica preconstruida- de la Educación Inclusiva expresan debilidades en su fuerza conceptualizadora, es decir, son incapaces de pensar analítica y metodológicamente a través de ellos, demostrando debilidades para hacer comprensible su objeto de estudio en su especificidad. Una aplicación que poseen los conceptos en el trabajo epistemológico, consiste en develar y delimitar el uso y asociación de determinados 
conceptos a una amplia multiplicidad de objetos a los que estos han sido aplicados. Si bien, en trabajos anteriores he focalizado mi interés en el estudio de las características de los elementos que integran el universo conceptual de la Educación Inclusiva, traducido en la naturaleza de su gramática -multiplicidad-, en sus condiciones de producción -viajeros y diaspóricos-, etc. En esta oportunidad, mi interés consiste en efectuar una aproximación inicial sobre los conceptos concebidos como herramientas epistemológicas que contribuyen a estudiar la Educación Inclusiva, es decir, qué tipo de aplicaciones metodológicas exigen o bien, adoptan determinados conceptos que permiten adentrarnos en el estudio de su campo de conocimiento. Si bien es cierto, no todos los conceptos - a pesar que pueden enlazarse en algún tópico o arista a su objetoposeen esta capacidad.

Algunos de ellos, poseen la capacidad de explorar el fenómeno, el campo y el objeto, determinan sus condiciones de enmarcamiento, al decir de Valderrama y De Mussy (2009), aquello que es legitimado y aceptado, o bien, rechazado. Los conceptos implicados en el sistema de enmarcamiento constituyen claves epistemológicas, delimitan con facilidad la especificidad de su estructura teórica y metodológica. El interés por los conceptos implicados en el 'estudio' de la Educación Inclusiva, sugiere la pregunta acerca de los modelos y las trayectorias analítico-metodológicas implicadas en su comprensión. Los conceptos, entonces, serán empleados como herramientas y modelos para el estudio del fenómeno y campo antes citado. Esta idea refuerza la afirmación de Bal (2002) al señalar que los conceptos pueden reemplazar a los métodos. Bachmann-Medick (2012) sugiere atender al conjunto de procesos mediación, mecanismos de transferencia y los particulares estilos de interacción que establecen entre sus objetos y formas comprensivas. En este apartado me propongo explorar las implicancias metodológicas del trabajo con conceptos específicamente aplicadas al estudio de la Educación Inclusiva, transitando hacia un examen acerca del propio concepto de inclusión, concluyendo acerca de las implicancias de la categoría de 'traducción', clave en la consolidación de un campo y de un objeto anti/post-disciplinar. ¿Qué implica trabajar con conceptos en el estudio de la Educación Inclusiva?, ¿qué otras categorías van complementando su comprensión?

El concepto de Educación Inclusiva es lecturado como un término contingente, flotante, complejo, dinámico e impreciso. 'Impreciso' producto de la mutabilidad 
constante de su objeto, el cual, se ha modificado, no ha si sus métodos de investigación y aproximación. Esta situación exige crear condiciones que permitan elegir adecuadamente sus significados, producto de las políticas de todo vale. Estos últimos, se enredan restringiendo la diferenciación entre el lenguaje teórico y metodológico. En tal caso, presenta un significado ambiguo y de amplio alcance, comúnmente utilizado como si su significado estuviese claro -producto del efecto de falsificación-, específicamente, en programas de investigación, políticas públicas y departamentos de formación del profesorado. Efecto de ello, consiste en la multiplicación de cursos de extensión, capacitación, de pre y post-graduación que refieren a Educación Inclusiva, sin evidenciar un departamento sobre esta temática ${ }^{21}$. Por todo ello, se observa un conjunto de desviaciones en torno a la institucionalización de su conocimiento. Epistemológicamente, el concepto y significado de la ‘inclusión' y la 'Educación Inclusiva' es esencialmente disputado por diversos campos del conocimiento -algunos con mayor o menor grado de proximidad-. Como 'concepto' expresa un carácter de intermediación, es decir, viaja y se moviliza por una amplia multiplicidad de disciplinas, recursos metodológicos y objetos de conocimientos heterogéneos entre sí. Observo la necesidad de efectuar un análisis más denso en torno a la diversidad de campos, objetos, influencias, métodos y teorías por las que este se moviliza, intentando identificar el tipo categoría -o bien en plural- de la cual emerge este sintagma. Producto de la complejidad que atraviesa al propio término y la incapacidad de los investigadores para aproximarse al fenómeno, particularmente, a su multidimensionalidad, es plausible afirmar que, su comprensión ha migrado penetrando en una multiplicidad de campos y discursos, exigiendo de esta forma, efectuar un mapa crítico $^{22}$ que dé cuenta de los diversos tipos de desplazamientos en los que ha devenido el término.

En múltiples campos el concepto de inclusión no aparece explícitamente como tal. Sin embargo, sus significados y propósitos intelectuales sustentan gran parte de la actividad científica de los Estudios Queer, los Estudios de Género, los Estudios de la Mujer, los Estudios sobre Feminismo Contemporáneo, e incluso, la corriente interseccional, los debates sobre Post-colonialidad y la Filosofía en su amplia gama de expresiones, tienden a reforzar estos objetivos en sus políticas de producción del

21 Véase: "La formación del profesorado y la comprensión epistemológica de la Educación Inclusiva: tensiones, permeabilidades y contingencias".

22 Véase: "Mapa Crítico de la Educación Inclusiva". 
conocimiento $^{23}$. Sin duda este punto es crucial, así como también lo son, el sentido y alcance de las discusiones que en ellos se albergan, demostrando que, aquellas que hoy consideramos parte central de las agendas de investigación sobre Educación Inclusiva, únicamente revisitan discusiones clásicas ampliamente desarrolladas por la mayoría de campos antes citados. Una amplia gama de disciplinas y discursos dedican una porción significativa de su actividad a puntos de captura significados como parte de la inclusión y, particularmente, de la Educación Inclusiva. Además, de aproximaciones situadas al abordaje del concepto.

La movilidad que atraviesa la categoría de inclusión distribuida por una multiplicidad de disciplinas, obligar a someter a traducción dichas contribuciones, a fin de ir ensamblando algo nuevo, un nuevo marco de especificación y conceptualización, nutrido de los aportes más significativos recopilados a lo largo de su viaje. Si bien es cierto, la categoría de traducción exige mayor especificación metodológica. Por todo ello, afirmaré que, el tratamiento del concepto de 'inclusión' y 'Educación Inclusiva' deviene en un concepto amplio y superpuesto. A objeto de evitar imponer análisis que conduzcan a nuevas condiciones de ambigüedad, es necesario orientar el trabajo con conceptos hacia el develamiento de las condiciones a las que pertenece el género de este objeto. Una de las dificultades metodológicas que podría enfrentarse al abordar su sentido y significado con ligereza, consistiría en la incapacidad para discutir su significado como problema académico, específicamente, cuando este, presenta un uso demasiado extendido. Caso específico del enfoque aquí discutido. Será entonces necesario puntualizar en la intención, el origen, el contexto y el contenido semántico (Bal, 2002). En su base, la Educación Inclusiva en tanto circunscripción intelectual expresa una serie de enredos genealógicos, nace específicamente de la categoría de multiplicidad, sustentando su actividad en la producción de lo nuevo ${ }^{24}$. ¿De qué categoría nace este concepto/sintagma?, ¿bajo qué mecanismos va desplazándose hacia otros campos dislocando sus fuerzas de conceptualización/producción? Por todo ello, afirmaré que, 'inclusión' y ‘Educación Inclusiva' es en sí mismo, un conjunto de viaje y un viaje epistemológico por el conocimiento educativo. Asignar este estatus a ambas nociones, sugiere atender cuya fuerza se desplaza más allá del nivel lingüístico y

\footnotetext{
${ }^{23}$ Explicita un concepto atravesado por complejas y diversas posicionalidades.

${ }^{24} \mathrm{Su}$ naturaleza lo define como un acontecimiento, irrumpe y esculpe la realidad, girando hacia rumbos imaginados.
} 
textual, emergiendo aplicaciones de usos metafóricos. Las metáforas en el estudio epistemológico aportan la capacidad de sustituir, remplazar y movilizar otras comprensiones vinculadas a un mismo fenómeno. Se encuentran estrechamente vinculadas a las condiciones tropológicas.

La creación de condiciones de fertilidad asociadas a la comprensión de los conceptos implicados en el Estudio de la Educación Inclusiva, sugiere la necesidad de someter a especificación metodológica la contribución de cada una de las disciplinas y otros recursos epistemológicos por las que esta se moviliza. En tal caso, la traducción desempeña un papel crucial, específicamente, en la especificación de un objeto genealógicamente anti/post-disciplinario. Inclusión y Educación Inclusiva son categorías que emergen en la intersección de una multiplicidad de disciplinas, discursos, influencias, marcos teóricos y políticos, métodos y objetos, etc. ¿De qué depende la potencialidad analítica de los conceptos implicados en el estudio de la Educación Inclusiva?, los conceptos como herramientas epistemológicas se convierten en dispositivos de focalización de su objeto. Los conceptos epistemológicos poseen la capacidad de entender la potencialidad de un determinado campo de conocimiento, resulta altamente fértil determinar algunos conceptos claves y determinar su función analítica, que no es lo mismo que ofrecer una lista de conceptos. ¿Qué conceptos nos ayudan a entender a comprender o estudiar la Educación Inclusiva en términos de ciencia anti/post-disciplinar?, ¿cuáles son las implicancias metodológicas y analíticas del trabajo en las intersecciones de las disciplinas?

El estudio de los conceptos - con una especificidad metodológica particular-al igual que los saberes de la Educación Inclusiva exige la creación de condiciones de traducción, el develamiento de la autenticidad de su campo emerge a través de diversas relaciones de traducción. En mi trabajo epistemológico la traducción se convierte en la búsqueda de condiciones de legibilidad de conceptos y saberes, devenida en una metodología -condición previa- de producción de saberes. Su propósito consiste en trasladar las condiciones de focalización de un determinando fenómeno hacia otras comprensiones. Considero oportuno demostrar la gran variedad de ámbitos de investigación que recurren y emplean los conceptos centrales en el estudio de la Educación Inclusiva. En este marco, las categorías de 'inclusión' y 'Educación Inclusiva' se convierten en nociones analíticas y acción. Mediante los conceptos es 
posible determinar los ejes de mutabilidad del objeto de la Educación Inclusiva y proponerse caminos para su abordaje metodológico.

Tal como he mencionado anteriormente, el concepto de Educación Inclusiva es en sí mismo un concepto de viaje, se mueve en una amplia multiplicidad de disciplinas, en encuadres culturales, en períodos históricos, entre comunidades académicas, zonas de contacto, consolidando un espacio diaspórico y fronterizo de producción. Consolida una unidad relacional de transferencia, captura, forclusión y re-articulación propiedades significativas en la configuración del objeto post-disciplinar de la misma. Consolida una unidad relacional de transferencia de teorías de diversas texturas, las que son reconfiguradas por ámbitos de producción, recepción y transferencia, identificando el conjunto de elementos olvidados, desplazados e incluso, rearticulados en su desarrollo. En consecuencia, la Educación Inclusiva deviene en una teoría itinerante. Coincido con Frank (2009) respecto de la potencialidad que ofrece el estudio de los movimientos de la Educación Inclusiva en su movilización por diversas disciplinas, pues, estos, nos ayudan a describir el conjunto de elementos que enriquecen su campo de producción. De modo que, los saberes, los conceptos, los objetos, las influencias y los recursos metodológicos que en dicha red analítica son albergados, no son estables, más bien son dinámicos, cambian constantemente. Sobre este particular, Bal (2002) y Bachmann-Medick (2012) sugieren inscribir el análisis en la identificación de sus rutas, métodos de desarrollo y mapas. El campo epistemológico de la Educación Inclusiva describe una espacialidad intelectual caracterizado por diversos tipos de movilidad, cada vez mayor en sus flujos de información de diversa naturaleza.

Cada campo de confluencia inaugura una modalidad de competencia disciplinaria específica y singular. En tanto, elemento constitutivo y pieza angular de su campo de conocimiento va configurando un pensamiento traslacional y fronterizo, producto de sus sistemas de mediación, se observa la capacidad de hacer surgir nuevos giros interpretativos y metodológicos. Su objeto de conocimiento emerge y se moviliza a través de una diversidad epistemológica, inaugurando una nueva forma de conocimiento. Como tal, no es concebido como algo restrictivo, forja nuevas formas de conocimiento, configura una categoría de análisis reflejada metodológicamente. De acuerdo con ello, es posible pensar la inclusión como un giro de traslación de los modos de producción y experimentación de la Ciencia Educativa. En este punto la 
'traducción' se convierte en un concepto clave en el estudio de la Educación Inclusiva, transformando y dislocando sus modos conceptuales, establece condiciones de legibilidad en torno a la capacidad extra-disciplinar de su campo de conocimiento, a través de él, se conectan campos alejados en su actividad científica, permitiendo depurar y extraer los aportes más significativos de cada disciplina, objeto, método, influencias, teorías, etc. confluentes en él. En tal caso, la traslación cobra relevancia en el trabajo en las intersecciones de las disciplinas, convirtiéndose en un recurso de (inter)mediación epistémico-metodológica. De esta forma, 'traslación', 'traducción’ e 'inclusión' se convierten en conceptos de carácter no-restrictivos, cuyas aplicaciones a diversos campos del conocimiento, da cuenta que el propio concepto de Educación Inclusiva se convierte en un concepto analítico referido a la examinación estructural sobre la heterotopicalidad de tensiones que en este campo convergen. El interés por los conceptos contribuye a la expansión del horizonte comprehensivo de la Educación Inclusiva.

Entre las tareas críticas que enfrenta la Educación Inclusiva destacan: a) la necesidad de visibilizar el llamado temprano de concebir la epistemología de la Educación Inclusiva como un sistema de actualización de la Teoría Educativa. Sobre este particular, es menester insistir en los elementos que permiten abordar el sentido y alcance de este enfoque como expresión de la transformación. b) Avanzar sobre el reconocimiento de la expansión que afecta a la categoría de inclusión concebida como modelo crucial en el estudio y análisis de la educación contemporánea y de sus principales problemáticas. La inclusión hoy, es un concepto complejo, flotante, compuesto por múltiples capas que generan constantemente diferencias analíticas, que conducen silenciosamente hacia la hibridez y a la confusión. d) la comprensión epistemológica de la Educación Inclusiva propiedad de la relación, el movimiento, la constelación y el encuentro de saberes, métodos, objetos, teorías, influencias, etc., cristaliza un tercer espacio -un no-lugar- trascendental para comprender la noción de inclusión como expresión de la transformación, una fuerza performativa -esculpe la realidad-que moviliza el cambio, siempre que este, exprese un compromiso social y político. De este modo, la comprensión epistemológica de la Educación Inclusiva se propone mapear críticamente, reorientando nociones previamente dominantes en el devenir de sus diversos campos y leyes del pensamiento enmarcadas en una 
periodización particular. Finalmente, los conceptos se convierten en recursos para un examen teórico más detallado. El estudio de los conceptos que explican analítica y metodológicamente la Educación Inclusiva ofrece una exploración específica acerca del conjunto de (inter)relaciones -de usos específicos y heterogéneos entre diversas clases de saberes, métodos, conceptos, objetos, teorías, influencias, etc.--, constituye un objetivo no resuelto al interior de su campo de producción, tampoco ajeno a polémicas y disputas conceptuales y metodológicas, así como, a categorías de descripción y análisis escasamente delimitadas. La inclusión se convierte en términos políticos y epistemológicos, en una estrategia de agenciamiento de la diferencia.

La práctica teórica y metodológica -abierta- de la Educación Inclusiva se forja a través de un conjunto de enredos genealógicos. La práctica conceptual por su parte, devela que muchas de sus categorías -contenidas en la noción de multiplicidad- se encuentran enredadas dentro y entre diferentes contextos de práctica política, analítica y teórica, enfoques, discursos, influencias y disciplinas académicas, preferentemente. Por consiguiente, deviene en un efecto de contaminación lingüística y abducción desmedida de conceptos. ¿De qué depende la confusión terminológica de la Educación Inclusiva? Para responder a esta interrogante, es necesario identificar el corpus de conceptos que desvirtúan la comprensión auténtica de su objeto. Sobre este particular, concuerdo con Bal (2002) y Bachmann-Medick (2012), respecto de la necesidad de describir analíticamente un conjunto de palabras clave, a fin de examinar y diferenciar exhaustivamente sus usos y mecanismos de aplicación a otros objetos y campos, demostrando sus estrategias de apropiación, evidenciando las razones, por las cuales, dicha acepción se ha convertido en un concepto relevante en la diversidad de saberes, métodos, conceptos, objetos, teorías, influencias, etc., confluyentes en este campo. Todo ello, exige la elaboración de una diferenciación cautelosa de cada término, previa determinación de las tradiciones a las que ha sido aplicada y el tipo de contextualismo que reclama su utilidad. Otra utilidad que expresa la descripción analítica de los conceptos que definen su universo conceptual, ha de ofrecer un sistema de diferenciación respecto de los términos relacionados que se usan para describir los encuentros y distanciamientos entre la diversidad de saberes, métodos, conceptos, objetos, teorías, influencias, etc. que participan de la configuración de su campo de conocimiento. Tales consideraciones permitirían superar los ejes que desenfocan el 
concepto de 'inclusión' y 'Educación Inclusiva', evitando asumir un término que conjuga un sinnúmero de errores de comprensión y aproximación a su objeto fenómeno- y método - conjunto de pasos para resolver algo-.

El uso actual del concepto de 'inclusión' y 'Educación Inclusiva' expresa un uso inflacionario, es decir, un incremento excesivo de un significado distorsionado. Para lo cual, es necesario develar la especificidad contextual de cada uno de sus conceptos configurantes. Al constituir la Educación Inclusiva un campo epistemológico de lo múltiple, es preciso, especificar el estudio de los conceptos a partir de sus funciones y tipologías. Para articular tal empresa, adscribiré a la propuesta efectuada por Bödeker (2009), entre los cuales, identifica: a) conceptos de lucha, b) conceptos de acción, c) conceptos de futuro, d) conceptos de perspectiva, e) conceptos de finalidad, f) conceptos de expectativas, g) conceptos de compensación, h) conceptos de integración, etc. Estas formas de organización facilitan la comprensión de determinados sentidos y significados. ¿Cuál es el papel que desempeñan los conceptos en la actividad científica de la Educación Inclusiva?: a) redefinición de categorías y significados, tanto en el campo fenomenológico como en el social, b) los conceptos organizan un grupo de fenómenos, c) determinan preguntas relevantes y c) determinan significados que pueden ser atribuidos al observador.

El universo conceptual de la Educación Inclusiva observa en su versión falsificada e hibridizada, deviene en un campo difuso, en la aplicación ligera e injustificada de ciertos conceptos, que, a su vez, carecen de estatus metodológico, es decir, carecen de capacidad de explicar o describir analíticamente un determinado fenómeno y sus ejes de especificidad. Su función queda reducida según Bal (2002) a una forma simple de nombrar. Si el examen de conceptos se efectúa en un terreno epistemológico que posee una estructura teórica clara, los conceptos fortalecen la comprensión de su objeto - a esto he denominado tropismo positivo- es decir, la producción de significantes y sus lógicas de estructuración giran en torno al núcleo auténtico del enfoque. De lo contrario, cuando existe una estructura teórica débil, preconstruida o bien, ininteligible, estos, actúan desvirtuando su objeto -hablamos entonces, de tropismo negativo-, es un giro que conduce a equívocos de interpretación. Los conceptos epistemológicos del enfoque aquí discutido, encierran una fuerza performativa, alterando la realidad y movilizando la transformación. No obstante, el 
performativo de la trasformación corre el riesgo de ser concebido como un performativo absoluto, pues carece de herramientas que organizan dicha empresa. La constitución performativa de los enunciados de la Educación Inclusiva alcanza su existencia en la ratificación de un compromiso social, lo que difiere de una simple afirmación a favor del cambio.

\section{4.-Conclusiones}

Los conceptos epistemológicos poseen la capacidad de predisponer la comprensión de determinados objetos, se convierten en herramientas que permiten valorar y describir críticamente a qué tipo de objetos han sido aplicados. Debido a la flexibilidad, movilidad y entropía que atraviesa al campo conceptual de la Educación Inclusiva es posible observar diversas prácticas de utilización de conceptos -reclaman la necesidad de develar su posición epistemológica- de forma desmedida, con cierta ligereza -propiedad del aplicacionismo epistemológico- dando paso a mecanismos de abducción y contaminación lingüística.

Los conceptos epistemológicos de la Educación Inclusiva son propiedades nolineales, articulan su actividad mediante un movimiento incesante a través de la multiplicidad de objetos, métodos, disciplinas y campos de conocimiento, preferentemente, cuyo funcionamiento queda determinado a través de la rearticulación de cada uno de ellos. A través de la metáfora del viaje propuesta por Bal (2002), es posible atender a las diversas formas de vinculaciones, afiliaciones, herencias epistemológicas, lingüísticas y metodológicas-. Su potencia ofrece la capacidad de describir mediante el establecimiento de un conjunto de distinciones analíticas, las principales características del objeto de la Educación Inclusiva y en particular, de la inscripción y niveles de comprensión de los denominados Estudios sobre Educación Inclusiva mediante múltiples sistemas de relación. La superposición de conceptos procedentes desde diversas disciplinas- constituye uno de los problemas más significativos, junto a los referidos a los de autenticidad. 


\section{Referencias Bibliográficas}

Bachmann-Medick, D. (2012). "Translation - A Concept and Model for the Study of Culture", en: Neumann, B., Nünning, A. (Edit.).Travelling Concepts for the Study of Culture. Germany: Hubert \& Co. GmbH \& Co. KG, Göttingen. Págs. 23-4.

Bal, M. (2002). Conceptos viajeros en las Humanidades. Una guía de Viaje. Málaga: Cendeac.

Bödeker, H.-E. (2009). Begriffsgeschichte, Diskursgeschichte, Metapherngeschichte. Göttingen: Wallestein Verlag.

De Mussy, L., Valderrama, M. (2009). Historiografía postmoderna: conceptos, figuras y manifiestos. Santiago: RIL/Universidad Finnis Terrae.

Frank, M. (2009). "Imaginative Geography as a Travelling Concept: Foucault, Said and the Spatial Turn". Recuperado el 26 de noviembre de 2018 de https://www.researchgate.net/publication/248914530_Imaginative_Geography_as_a_Tr avelling_Concept_Foucault_Said_and the Spatial_Turn

Knorr, K. (2005). La fabricación del conocimiento. Un ensayo sobre el carácter constructivista y contextual de la ciencia. Buenos Aires: Ed. Univ. Nacional de Quilmes.

Mowitt, J. (1992). Text: The Genealogy of an Antidisciplinary Object. Durham and London: Duke.

Ocampo, A. (2018a). La formación del profesorado y la comprensión epistemológica de la Educación Inclusiva: tensiones, permeabilidades y contingencias. Santiago: Fondo Editorial CELEI.

Ocampo, A. (2018b). "Conceptos Viajeros en Educación Inclusiva y sus tareas críticas: tensiones analítico-metodológicas", en: Román, E. (Eds.). Escenarios Educativos Latinoamericanos. Una mirada desde la Universidad. (pp.295-329). La Habana: REED.

Ocampo, A. (2019). Mapa crítico de la educación inclusiva. Santiago: Fondo Editorial CELEI.

Richard, N. (2009). “Antidisciplina, Transdisciplina y Redisciplinamientos del Saber". Recuperado el 16 de noviembre de 2018 de http://ediciones.ucsh.cl/ojs/index.php/TSUCSH/article/view/185

Stengers, I. (1994). Entre el tiempo y la eternidad. Madrid: Alianza Editorial. 